\title{
Enfermedad de Kawasaki: A propósito de un caso atípico y con intususcepción.
}

\author{
TORI TORI Carlos*, BAZÁN ZENDER Carlos**, VARGAS GALGANI Mario.***
}

\section{SUMMARY}

We present a rare association of atypical Kawasaki disease and intussusception in a three month old male patient. It all began with high fever and an obstructive intestinal syndrome developed in the second day of hospitalization, diagnosed as a colo-colonic intussusception. Despite an adecuate postoperative antibiotic coverage the high fever continued, which made us pursue a diagnosis of a vasculitis syndrome among other entities. The echocardiogram showed dilatation of both coronary arteries. The currently accepted clinical criteria for Kawasaki disease may not always allow its identification in patients who are younger than six months of age. A diagnosis of Kawasaki disease and an echocardiographic evaluation of the coronary arteries should be considered in young infants with prolongued fever of unkown origin. ( Rev Med Hered 2001; 12: 37-41).

KEY WORDS: Kawasaki, mucocutaneous lymph node syndrome, atypical vasculitis, intususception, children.

\section{INTRODUCCIÓN}

Presentamos el caso de un paciente varón de tres meses de edad con enfermedad de Kawasaki atípica e intususcepción, lo cual constituye una asociación infrecuente. La continuidad de la fiebre a pesar de una cobertura antibiótica adecuada, la ausencia de otra sintomatología y la dilatación de ambas arterias coronarias en el ecocardiograma nos llevaron al diagnóstico de enfermedad de Kawasaki atípica.

\section{Enfermedad Actual}

A.D.S., varón de tres meses de edad con fiebre de 36 horas de evolución e irritabilidad, pero en buen estado general. Un día antes de su ingreso había concurrido al
Servicio de Emergencia por llanto frecuente y fiebre. Al día siguiente acude nuevamente a Emergencia por fiebre alta y ningún otro síntoma. El examen físico era normal, no habiendo adenopatías,signos meníngeos, ni hepato o esplenomegalia.

Antecedentes familiares: Asma bronquial, diabetes mellitus y lupus eritematoso en la rama materna.

Como la fiebre era bastante alta, se hospitalizó al paciente y se inició tratamiento con ceftriaxona y gentamicina por vía endovenosa.

El hemograma mostró anemia leve, leucocitosis y trombocitosis (Cuadro $\mathrm{N}^{\circ} 1$ ). Las transaminasas y el examen de orina fueron normales. Los cultivos fueron

\footnotetext{
* Pediatra, Clínica San Felipe. Profesor Invitado del Departamento de Pediatría, Universidad Peruana Cayetano Heredia. Lima, Perú.

** $\quad$ Cirujano-Pediatra, Clínica San Felipe. Lima, Perú.

*** Médico-Cardiólogo, Clínica San Felipe. Lima, Perú.
} 


\section{CUADRO N ${ }^{0} 1$}

\begin{tabular}{|c|c|c|c|c|c|}
\hline & & & $\operatorname{ro} \mathrm{N}^{\mathrm{O}} 1$ & & \\
\hline & 06-Feb-01 & 07-Feb-01 & 09-Feb-01 & 11-Feb-01 & $\begin{array}{c}\text { 15-Feb-01 } \\
\text { Post-IGG }\end{array}$ \\
\hline $\begin{array}{l}\text { Hemoglobina } \\
\mathrm{g} / 100 \mathrm{ml}\end{array}$ & 10.2 & 9.9 & 10.3 & 10.4 & 13.0 \\
\hline Hematocrito & 29 & 28.3 & 30.6 & 31.5 & 39.2 \\
\hline Hematies X mm3 & $3^{\prime} 790,000$ & $3^{\prime} 700,000$ & $33^{\prime} 910,000$ & $3^{\prime} 890,000$ & $4^{\prime} 900,000$ \\
\hline Leucocitos $\mathrm{Xmm3}$ & 31,600 & 14,200 & 17,700 & 19,800 & 10,900 \\
\hline Mielocitos & 0 & 0 & 0 & 0 & 0 \\
\hline Metamielocitos & 0 & 0 & 0 & 0 & 0 \\
\hline Abastonados & 1 & 3 & 4 & 3 & 0 \\
\hline Segmentados & 70 & 59 & 48 & 49 & 39 \\
\hline Linfocitos & 23 & 34 & 45 & 40 & 51 \\
\hline Monocitos & 5 & 2 & 0 & 4 & 2 \\
\hline Eosinófilos & 1 & 2 & 3 & 4 & 7 \\
\hline Basófilos & 0 & 0 & 0 & 0 & 1 \\
\hline Plaquetas $\mathrm{Xmm3}$ & 897000 & 569000 & 538000 & 530000 & 697000 \\
\hline
\end{tabular}

negativos.

Al día siguiente la fiebre siguió alta, y se notó cierta resistencia a la palpación del hemiabdomen derecho. El estudio radiográfico de abdomen reveló obstrucción intestinal cuya causa fue una intususcepción colo-colónica a la altura del ángulo esplénico del colon. En el postoperatorio inmediato se reemplazó la gentamicina por aztreonam y se agregó metronidazol parenteral. Al segundo día postoperatorio se notaron los labios secos con lesiones costrosas.

El paciente siguió con fiebre alta hasta de $41^{\circ} \mathrm{C}$ du- rante los cinco días postoperatorios a pesar de una buena cobertura antibiótica. El ecocardiograma mostró dilatación de las arterias coronarias en su trechos proximales con 3 y $4 \mathrm{~mm}$ de diámetro ( ver Fotos 1 y 2 ).

El paciente recibió ese mismo día inmunoglobulina endovenosa, $2 \mathrm{gr} / \mathrm{Kg}$ de peso, y aspirina $80 \mathrm{mg} / \mathrm{Kg}$ de peso al día por vía oral, administrada cada 6 horas y ranitidina .

La respuesta fue inmediata. Al término de la administración de la inmunoglobulina, la fiebre bajó y el paciente permaneció afebril. La dosis alta de aspirina se mantuvo durante catorce días y luego se disminuyó a $5 \mathrm{mg} / \mathrm{Kg}$ al día y permanecerá así durante tres meses.

El ecocardiograma efectuado 10 días después del primero mostró una reducción del $50 \%$ en el calibre de las arterias coronarias.

\section{DISCUSION}

La enfermedad de Kawasaki consiste en una vasculitis multisistémica aguda, febril y autolimitada que afecta casi exclusivamente a los niños en edad pre-escolar (1). Esta enfermedad fue descrita originalmente por Kawasaki en la literatura japonesa el año 1967, a la cual denominó Sindrome Mucocutáneo Linfático atribuyéndole un curso benigno (2).

La edad pico de presentación es entre el primer y

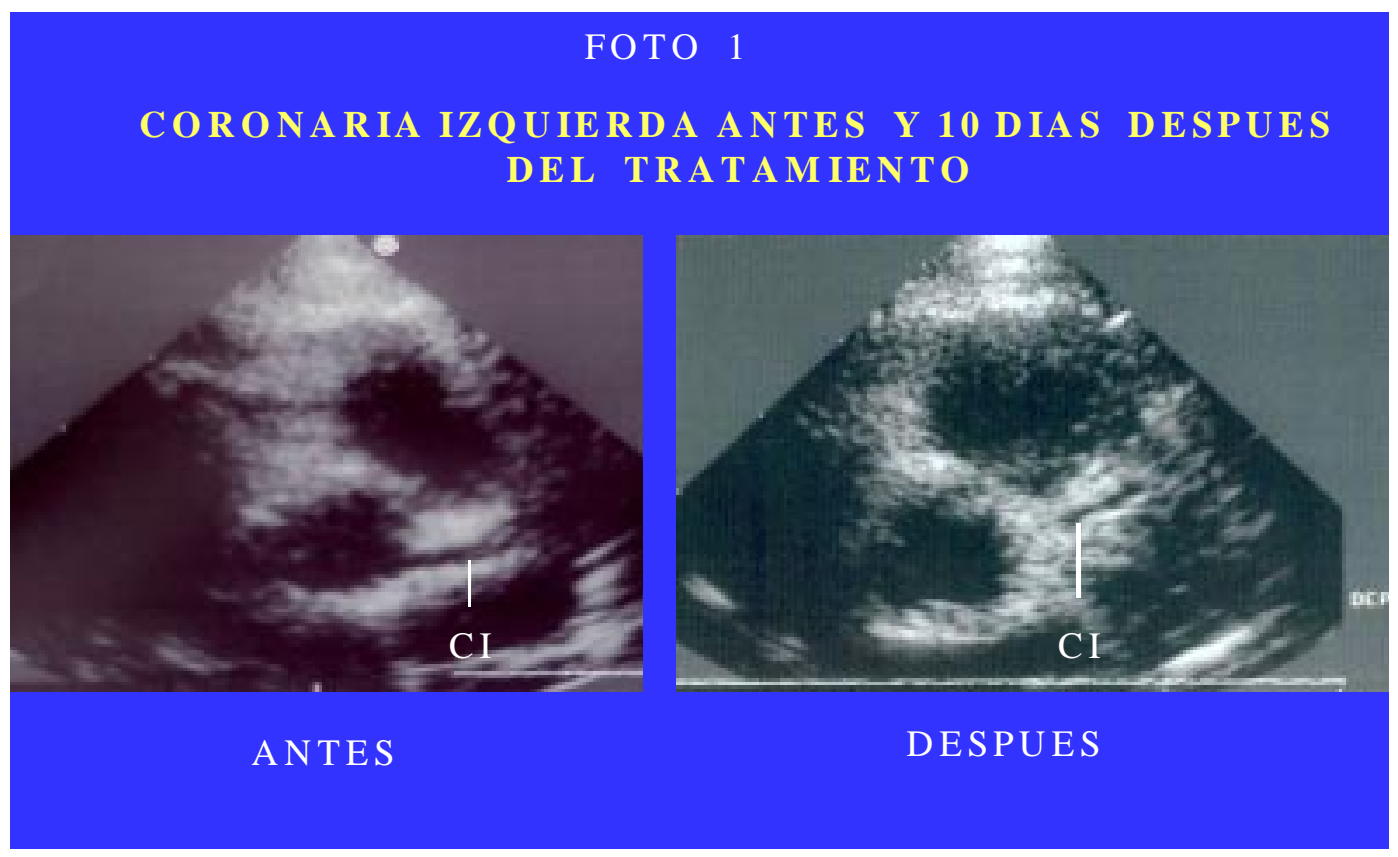


segundo año de vida, con el $80 \%$ de los casos en menores de 4 años de edad. Los varones son más afectados que las mujeres en una proporción de 1.5 a 1 . Individuos de raza japonesa y coreana tienen un mayor riesgo de enfermedad (1,3,4). En los países desarrollados la enfermedad de Kawasaki ha reemplazado a la fiebre reumática como la causa más común de enfermedad cardiaca adquirida en pediatría (5).

La enfermedad de Kawasaki es una enfermedad trifásica de inicio agudo con fiebre alta como primer signo y una duración de 7 a 14 días. Se acompaña de inyección conjuntival sin secreción ocular, cambios en la mucosa oral como eritema de faringe, lengua aframbuesada, labios fisurados; un edema indurado y coloración purpúrea de palmas y planta de los pies; un rash máculo-papular o morbiliforme principalmente en el tronco, y linfadenopatía cervical con ganglios de diámetro no mayor de $1.5 \mathrm{~cm}$. Le sigue una fase subaguda de 25 días de duración, durante la cual desaparecen la fiebre, el rash y la linfadenopatía y se agregan descamación de los dedos, artralgias, artritis y disfunción miocárdica.

La fase de convalescencia se inicia cuando ya no hay signos clínicos y continúa hasta que la velocidad de sedimentación retorna a lo normal, usualmente a los 70 días de iniciada la enfermedad.

Aunque los niños menores de 6 semanas de edad pueden tener los signos clásicos de la enfermedad, el diagnóstico en los primeros tres meses de vida es infrecuente, por presentar ellos manifestaciones leves o atípicas, con menos signos diagnósticos o sólo fiebre y el hallazgo de anomalías en las arterias coronarias, como en el caso presente $(6,7,8)$. Esto es lo que constituye el Kawasaki atípico.

Estas anormalidades en las coronarias se comienzan a visualizar entre el final de la primera semana y comienzos de la segunda. Consisten en dilatación progresiva de las arterias coronarias, alcanzando mayor incidencia y severidad al mes de su inicio. En total, un 18 a $25 \%$ de pacientes van a desarrollar estos aneurismas, y los varones menores de 1 año son los que están en mayor riesgo. Otros hallazgos cardiovasculares son: pericarditis $(30 \%)$, ritmo de galope, insuficiencia cardiaca $(20 \%)$, insuficiencia tricuspídea $(30 \%)$, insuficiencia mitral (25\%), miocarditis, y bloqueo cardíaco de primer grado (1). Además pueden presentar meningitis aséptica, artralgias y artritis, distensión aguda de la vesícula biliar, diarrea (25\%), hepatopatía (40\%), neumonitis , otitis media, uretritis $(60 \%)(1)$, anemia hemolítica autoinmune (9), psoriasis (9), hipertensión renovascular (10), colitis izquierda (11) e isquemia periférica severa resultante en gangrena (12). Otro signo es el eritema e induración en el sitio de inoculación del bacilo de Calmette-Guerin (13).

Los resultados de exámenes de laboratorio son una muestra del grado de imflamación sistémica: leucocitosis, algunas veces con desviación izquierda; anemia; aumento de la velocidad de sedimentación; presencia de proteína $\mathrm{C}$ reactiva, de alfa-1-antitripsina $\mathrm{y}$, también, trombocitosis. Nuestro paciente mostró una leucocitosis importante, anemia y trombocitosis.

La intususcepción es un cuadro bastante infrecuente en pacientes con Enfermedad de Kawasaki. Solamente hay un caso descrito en la literatura en un bebe de sexo femenino de 4 meses de edad (14). Esto es de llamar la atención, ya que hay casos de intususcepción descritos en otros tipos de vasculitis como en la púrpura de Henoch-Schönlein, en el edema hemorrágico agudo que es una forma de vasculitis leucocitoclástica limitada a la piel, y en el lupus eritematoso diseminado $(15,16,17,18,19)$.

En cuanto al tratamiento, la respuesta a la inmunoglobulina endovenosa y aspirina en altas dosis es bastante rápida. Dos tercios de los pacientes permanecerán afebriles y mejorados después de 24 horas de haber completado el tratamiento endovenoso. El $90 \%$ lo estará a las 48 horas. Aquellos que permanecen con fiebre tienen un alto riesgo de desarrollar anomalías coronarias (1).

La administración de inmunoglobulina endovenosa en la fase aguda de la enfermedad reduce la prevalencia de dilatación coronaria a menos del $5 \%$ y la de aneurismas coronarios gigantes a menos del $1 \%$ (20).

El pronóstico de esta enfermedad empeora si existen factores como el ser de sexo masculino, tener menos de 1 año de edad, haber tenido fiebre por lo menos 14 días o recrudecencia de la fiebre, tener una hemoglobina menor de $10 \mathrm{gm} / \mathrm{dl}$, leucocitosis de más de 30,000/ $\mathrm{mm} 3$, elevación persistente de la proteina C reactiva y una velocidad de sedimentación mayor de $30 \mathrm{~mm} /$ hora (Westergren)(21).

La trombocitopenia está asociada con el desarrollo de enfermedad coronaria severa e infarto de miocardio (22).

En conclusión, la Enfermedad de Kawasaki debe considerarse siempre en el diagnóstico diferencial de una fiebre prolongada en niños. Ocasionalmente la fiebre puede ser la única manifestación de la enfermedad en pacientes menores de seis meses de edad. En tales 


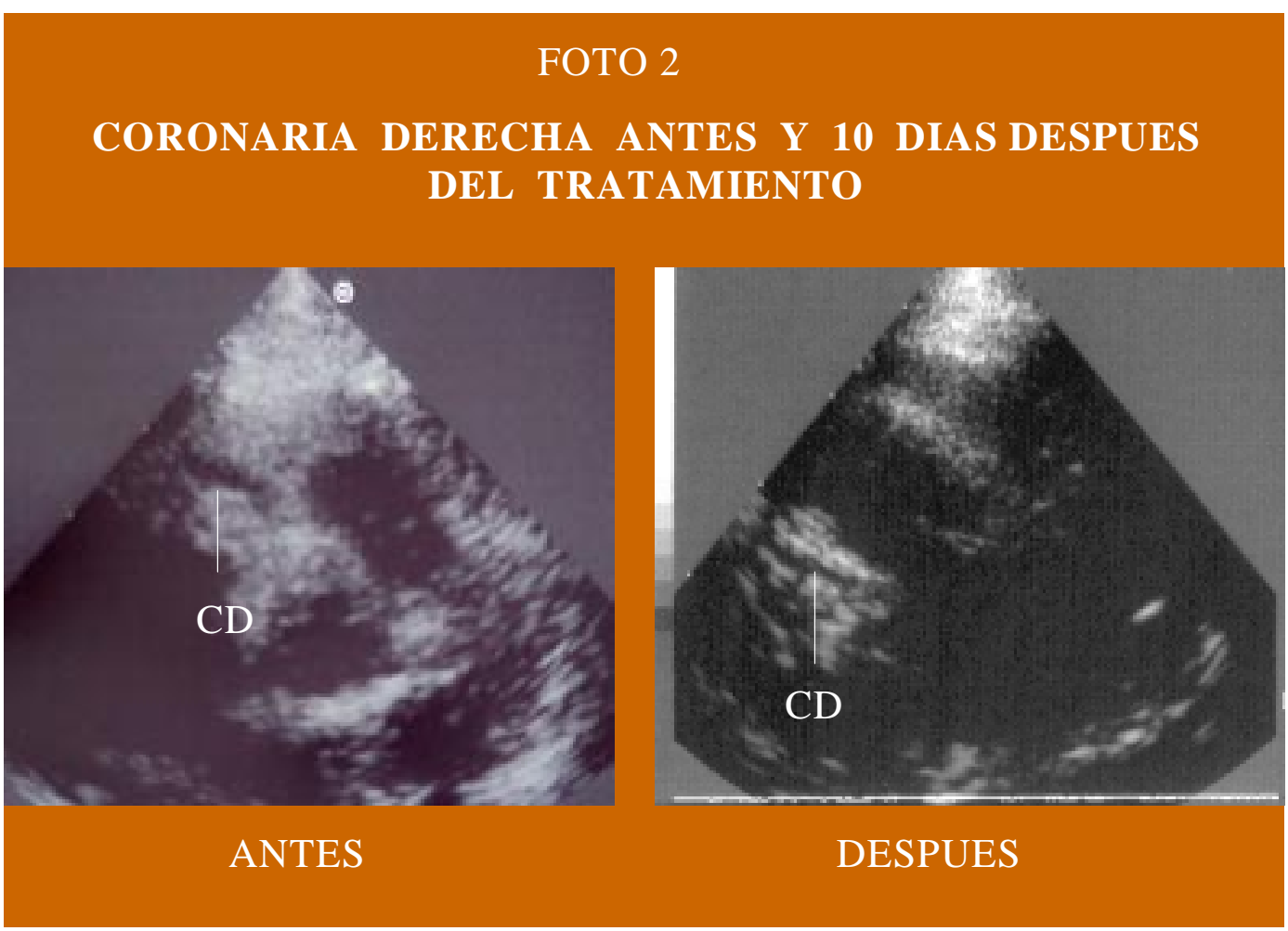

casos, el diagnóstico se confirma con el hallazgo de aneurismas coronarios en el ecocardiograma (23). Seguir exactamente los criterios actualmente aceptados para diagnosticar la enfermedad de Kawasaki puede llevarnos a no reconocer sus formas incompletas, con secuelas potenciales de infarto de miocardio y muerte súbita (8).

\section{Correspondencia}

Dr. Carlos Tori Tori.

Clínica San Felipe. Av. Gregorio Escobedo 660, Of 404. Lima 11, Perú.

\section{REFERENCIAS BIBLIOGRAFICAS}

1. Melish ME. Kawasaki Syndrome. Pediatrics in Review 1996; 17(5):153-162.

2. Kawasaki T: Acute febrile mucocutaneous syndrome with lymphoid involvement with specific desquamation of the fingers and toes in children [Japanese]. Jpn J Allergy 1967; 16:178-222.

3. Morens DM, Anderson LJ, Hurwitz ES. National surveillance of Kawasaki disease. Pediatrics 1980; 65:21-5.

4. Dean AG, Melish ME, Hicks R, et al: An epidemic of Kawasaki syndrome in Hawaii. J Pediatr 1982; 100:552557.

5. Taubert KA, Rowley AH, Shulman ST: A 10 year (19841993) United States hospital survey of Kawasaki disease. In Kato H (ed): Kawasaki Disease. Elsevier Sci- ence, Amsterdam, The Netherlands, 1995, pp 34-38

6. Boven K, DeGraeff-Meeder ER, Spliet W, et al: Atypical Kawasaki disease: An often missed diagnosis. Eur J Pediatr 1992; 151:577-580.

7. Burns JC, Wiggins JW, Toews WH, et al: Clinical spectrum of Kawasaki disease in infants younger than 6 months of age. J Pediatr 1986;109:759-763.

8. Rowley AH, Gonzalez-Crussi F, Gidding SS, et al: Incomplete Kawasaki disease with coronary artery involvement. J Pediatr 1987; 110:409-413.

9. Burke AP, Virmani R, Perry LW, Ling Li, King TM, Smialek J. Pediatrics 1998; 101:108-111.

10.Foster BJ. Kawasaki disease complicated by renal artery stenosis. Arch Dis Child 2000; 83: 253-5.

11.Chung CJ. Kawasaki disease presenting as focal colitis. Pediatr Radiol 1996; 26:455-7.

12.Tomita S, Chung K, Mas M, et al: Peripheral gangrene associated with Kawasaki disease. Clin Infect Dis 1992; 14:121-126.

13.Suzuki A, Yamagishi M, Kimura K, et al: Functional behavior and morphology of the coronary artery wall in patients with Kawasaki disease assessed by intravascular ultrasound. J Am Coll Cardiol 1996; 27:291-296.

14.Huang YC.Unusual manifestations in children with Kawasaki disease. J Formos Med Assoc 1997; 96: 451-6.

15.Robson WL, Leung AK: Henoch-Schonlein purpura. Adv Pediatr 1994; 41:163-194.

16.Lanzkowsky S, Lanzkowsky L, Lanzkowsky P: HenochSchoenlein purpura. Pediatr Rev 1992; 13:130.

17.Roberti I, Reisman L, Churg J: Vasculitis in childhood. Pediatr Nephrol 1993; 7:479.

18.Baselga E, Drolet BA,Esterly NB. Purpura in infants and 
children. Journal of the American Academy of Dermatology 1997; 37:673-708.

19.Bailey M, Chapin W, Licht H, Reynolds JC. Gastrointestinal Disorders and Systemic Disease, Part II. The Effects of Vasculitis on the Gastrointestinal Tract and Liver. Gastroenterology Clinics 1998; 27:747-782.

20.Newburger JW. Kawasaki disease: Who is at risk? J Pediatr 2000;137:149-52.

21. Asai T. [Diagnosis and prognosis of coronary artery le- sions in Kawasaki disease. Coronary angiography and the conditions for its application (a score chart)]. Nippon Rinsho 1983;41:2080-5. (Jpn).

22.Niwa K, Aotsuka H, Hamada H, et al: Thrombocytopenia: A risk factor for acute myocardial infarction during the acute phase of Kawasaki disease. Coron Artery Dis 1995; 6:857-864.

23. Rowley AH, Shulman ST. Kawasaki Syndrome. Pediatr Clin North Am 1999; 46:313-329. 